\title{
Detection of Serum Allergen-Specific IgE in Atopic Dogs Tested in Northern Italy: Preliminary Study
}

\author{
Morena Di Tommaso ${ }^{1, *(\mathbb{D} \text {, Alessia Luciani }}{ }^{1}$, Paolo Emidio Crisi ${ }^{1, *}$, Marica Beschi ${ }^{2}$, Paolo Rosi ${ }^{2}$, \\ Francesca Rocconi ${ }^{1,+}$ and Arianna Miglio ${ }^{1,+}(\mathbb{D}$ \\ 1 Faculty of Veterinary Medicine, Veterinary University Hospital, University of Teramo, Piano d'Accio, \\ 64100 Teramo, Italy; aluciani@unite.it (A.L.); frocconi@unite.it (F.R.); amiglio@unite.it (A.M.) \\ 2 Veterinary Clinic Dr. Paolo Rosi, via Brescia 46, 25086 Rezzato (BS), Italy; marica.beschi@gmail.com (M.B.); \\ clinicaveterinariadott.rosi@gmail.com (P.R.) \\ * Correspondence: mditommaso@unite.it (M.D.T.); pecrisi@unite.it (P.E.C.) \\ + These authors contributed equally to the work.
}

Citation: Di Tommaso, M.; Luciani, A.; Crisi, P.E.; Beschi, M.; Rosi, P.; Rocconi, F.; Miglio, A. Detection of Serum Allergen-Specific IgE in Atopic Dogs Tested in Northern Italy: Preliminary Study. Animals 2021, 11, 358. https://doi.org/10.3390/ani 11020358

Academic Editor: Simon L. Priestnall Received: 31 December 2020

Accepted: 27 January 2021

Published: 1 February 2021

Publisher's Note: MDPI stays neutral with regard to jurisdictional claims in published maps and institutional affiliations.

Copyright: (c) 2021 by the authors Licensee MDPI, Basel, Switzerland. This article is an open access article distributed under the terms and conditions of the Creative Commons Attribution (CC BY) license (https:// creativecommons.org/licenses/by/ $4.0 /)$.
Simple Summary: Canine atopic dermatitis (CAD) is a genetically predisposed allergic skin disease associated to IgE-mediated hypersensitivity. Serum Allergen-Specific IgE test (SAT) is used to detect allergen-specific IgE antibodies and to set-up a specific immunotherapy. The present study aims to identify the most relevant environmental allergens in a population of atopic dogs living in Northern Italy by using SAT, due to the absence of data in this area. Out of 117 selected client-owned dogs, 69 were included in the study. A screening test was used to identify indoor and outdoor allergens positivity and a specific serum allergy test was performed to detect an extended panel of allergens. Among the 49 positives to the screening test, $53 \%$ were positive for both indoor and outdoor, $38.8 \%$ only for indoor, and $8.2 \%$ only for outdoor allergens. Mites and pollen of Rumex acetosa, and grasses were the most represented allergens. This is the first study to collect data on the frequency of specific allergens involved in CAD in Italy by using SAT. In accordance with the result of this study, specific panels for geographical areas should be considered and re-evaluated at time intervals since numerous factors affect the prevalence of IgE positivity in atopic dogs.

Abstract: Canine atopic dermatitis (CAD) is a pruritic allergic skin disease associated with IgEmediated hypersensitivity. IgE is detected using Serum Allergen-Specific IgE test (SAT) in order to identify allergens. The present study aims to identify the environmental allergens in atopic dogs living in Northern Italy using SAT. The screening SAT (sSAT), using a monoclonal antibody cocktailbased ELISA to identify indoor and outdoor allergens, was performed. In all positive samples, an anti-IgE monoclonal antibody ELISA test was performed to extend panel of allergens. Out of 117 selected dogs, 69 were included in the study; $71 \%$ were positive and $29 \%$ were negative to sSAT. Among the 49 positive sSAT, 53\% were positive for both indoor and outdoor, $38.8 \%$ only for indoor, and $8.2 \%$ only for outdoor allergens. This is the first study on the frequency of allergens involved in CAD in Italy using SAT. IgE hypersensitivity in atopic dogs of Northern Italy is usually associated with indoor allergens, primarily house dust mites. Among the outdoor allergens, an important role was played by Rumex acetosa. Polysensitization also commonly occurs. Therefore, since the numerous factors affect the IgE positivity in CAD, specific panels for geographical areas should be considered and re-evaluated at time intervals.

Keywords: dog; atopic dermatitis; IgE; serology; allergy testing; Northern Italy

\section{Introduction}

Canine atopic dermatitis (CAD) is a genetically predisposed inflammatory and pruritic allergic common skin disease with characteristic clinical features associated with IgE-mediated hypersensitivity [1-3]. These antibodies are most commonly directed against environmental allergens, such as mite antigens, house dust, epidermal antigen, insect antigens, pollens, and 
mold spores $[1,2,4,5]$. Climate, vegetation, lifestyle, population density, and pollution levels are all factors that might contribute to the varying occurrence of CAD [6].

The diagnosis of CAD is based on history, clinical signs, and exclusion of other pruritic diseases [3]. The presence of allergen-specific IgE detected by Intradermal Test (IDT) or by Serum Allergen-Specific IgE test (SAT) is not useful for diagnosis due to the low specificity and sensitivity of these tests $[7,8]$. They do not have a good ability to discriminate between atopic and non-atopic patients [3]. In addition, in some dogs, clinical signs of CAD are present, even in the absence of detectable IgE (CAD-like) [4]. Only after clinical diagnosis of CAD is achieved, should IDT and/or SAT be used to identify the responsible allergen and to set up a specific immunotherapy [3,9]. Until recent years, IDT was the most commonly used method by dermatologists, however it is not of practical use for dogs and cats as patients need sedation and single inoculations of individual allergen extracts. In addition, numerous drug interferences have been demonstrated [10,11]. On the other hand, SAT is today widely used in clinical practice $[10,11]$ due to the lack of risk and discomfort for the patient, the detection of multiple antigens by using a single serum sample, the quantitative nature of the results, the fact that the test can be performed on patients in the presence of a widespread cutaneous inflammation, and because it is less affected by of current drug therapies [12-14]. A serologic test is also required for large-scale investigations of CAD [15]. Since causative allergens vary according to geographical region, climate, environmental pollution/hygiene, and residential environments [15], knowledge of the geographic distribution of allergens is very important to set-up a panel targeted for a specific geographical area [16].

There are various studies from different countries around the world that investigate the prevalence of positive reactions to different aero-allergens in atopic dogs $[6,15,17-26]$. However, there are only a few studies on the distribution of allergens in Italian regions $[27,28]$.

The present study aims to identify the most relevant environmental allergens in a population of atopic dogs living in Northern Italy by evaluating the seropositivity to allergen-specific IgE by using monoclonal antibody based enzyme-linked immunosorbent assay, due to the lack of data in this geographical area.

\section{Materials and Methods}

This study was carried out from May 2016 to October 2019, during different seasons and at the Veterinary private Clinic located in Brescia (Northern Italy). All animals tested were living in Northern Italy.

\subsection{Criteria for Animal Selection}

One hundred and seventeen client-owned dogs with clinical diagnosis of CAD were selected for the study. Diagnosis of CAD was made on the basis of history, the presence of characteristic clinical signs, i.e., at least 5 of 8 clinical features of CAD as described by Favrot et al. [29] (Table 1), and by excluding other skin condition with similar clinical signs and/or pruritic skin diseases that can resemble or overlap with CAD $[3,7,29]$. The administration of drugs such as glucocorticoids, oclacitinib, lokivetmab, antihistamines, and cyclosporine were discontinued at least 4 weeks before enrolment in the study.

Table 1. Favrot's criteria. Set 1 (use for clinical studies).

\begin{tabular}{cc}
\hline No. & Criteria \\
\hline 1 & Age at onset $<3$ years \\
2 & Mostly indoor \\
3 & Corticosteroid-responsive pruritus \\
4 & Chronic or recurrent yeast infections \\
5 & Affected front feet \\
6 & Affected ear pinnae \\
7 & Non-affected ear margins \\
8 & Non affected dorso-lumbar area \\
\hline
\end{tabular}




\subsection{Exclusion Criteria}

Dogs with other pruritic skin diseases such as dermatitis due to Malassezia, superficial pyoderma, fungal skin infection, ectoparasitic, and neoplastic skin diseases were excluded from the study by performing appropriate diagnostic tests (skin cytology, multiple skin scrapings, hair plucking, hair combing, acetate tape impressions, ear swabbing, fungal culture) [7]. A strict elimination diet trial with commercial hydrolyzed protein was administered for at least 8 weeks prior to testing and an antiparasitic trial treatment was applied to rule out food allergy dermatitis and ectoparasite skin disease, respectively [7]. Subjects with $\mathrm{CAD}$ and concurrent conditions that needed treatment were excluded from the study.

\subsection{Sample Collection and Analysis}

Serum samples were collected and shipped on the same day to a reference laboratory (Univet Diagnostic Services, Barcelona, Spain) to perform SAT using monoclonal antibody cocktail-based enzyme-linked immunosorbent assay (macELISA, Greer Laboratories, Lenoir, NC, USA). This followed a previously well-established procedure [30,31] used to evaluate allergen-specific IgE in serum samples.

An anti-IgE macELISA screening serum allergy test (sSAT) was initially performed in order to identify the negativity or the positivity to significant levels of IgE against two categories: Indoor and outdoor allergens. The total number of allergens tested in each animal was 25 (10 indoor and 15 outdoor allergens), as is commonly used in European laboratories. The composite of the indoor allergen panel (IN) consisted of a mixture of 2 house dust mites, 3 storage mites, 3 molds, Malassezia, and flea antigens. The composite of the outdoor allergen panel (OUT) consisted of a mixture of pollen from 5 grasses, 6 weeds, and 4 common tree allergens. All results were expressed as ELISA Absorbance Units (EAU) and a cut-off of 150 EAU was established [30,31].

Subsequently, to identify the composition of allergen-specific immunotherapy, in all positive samples to IN and/or OUT an anti-IgE monoclonal antibody ELISA specific serum allergy test (UNITEST-ELISA, Greer Laboratories, Lenoir, NC, USA) was performed in the same laboratory. This detected an extended panel of allergens in the dogs:

- IN serum allergen-specific IgE tests: Dermatophagoides farinae, Dermatophagoides pteronyssinus, Acarus siro, Tyrophagus putrescentiae, Lepidogliphus destructor, Alternaria alternata, Aspergillus fumigatus, Penicillium, Malassezia, and fleas.

- OUT serum allergen-specific IgE tests: Phleum pretense, Dactylis glomerata, Poa pratendis, Lolium perenne, Cynodon dactylon, Taraxacum vulgare, Artemisia vulgaris, Rumex acetosa, Plantago lanceolata, Parietaria spp., Chenopodium álbum, Platanus acerifolia, Olea europaea, Betula pendula, and Cupressus sempervirens.

\subsection{Statistical Analysis}

Data analysis was performed using statistical software packages (MedCalc 17.9.2, MedCalc Software, Mariakerke, Belgium). All data were evaluated using a standard descriptive statistic. Normality was checked using the D'Agostino-Pearson test. A comparison between groups was done using the unpaired $t$ test or the Mann-Whitney test, based on their distribution. For the statistical analyses, a $p$ value $<0.05$ was considered significant.

\section{Results}

Based on exclusion criteria, 48 dogs were rule out. Out of 69 atopic dogs included in the study, $44(63.8 \%)$ were males and $25(36.2 \%)$ were females. These subjects were aged between 2 and 10 years (median of 5 years). Various breeds were represented, such as Labrador Retriever $(n=11)$, French Bulldog $(n=10)$, German Shepherd dog $(n=8)$, American Staffordshire Terrier $(n=6)$, Mixed breed $(n=6)$, English Bulldog $(n=5)$, Rottweiler $(n=4)$, Bernese Mountain dog $(n=3)$, Shih Tzu $(n=2)$, Chihuahua $(n=2)$, and one subject for each of the following dog breeds: Yorkshire Terrier, Shar Pei, Weimaraner, Pug, Border Collie, Chow Chow, Dalmatian, Australian Shepherd dog, Akita Inu, Staffordshire Bull Terrier, American Pitt Bull Terrier, and Scottish Terrier. 
Of the 69 screening tests performed, 49 were positive $(71 \%)$ and 20 were negative $(29 \%)$. Among the 49 positive screening tests, 26 were positive for both IN and OUT (53\%), 19 were positive only for IN $(38.8 \%)$, and 4 were positive only for OUT $(8.2 \%)$. There were no significant differences between serum IgE levels of allergens in IN positive subjects compared to OUT positive subjects $(p=0.17)$.

All IN positive subjects $(n=45,91.8 \%)$ showed a positivity for at least one allergen of the mite family, 12 for at least one allergen in the mold category, 11 for the Malassezia, and 1 for the flea saliva allergen. Serum IgE concentrations and distribution of various allergens in IN positive subjects is reported in Table 2 and Figure 1, respectively.

Table 2. Serum IgE concentrations (ELISA Absorbance Units (EAU)) of various allergens in IN positive subjects $(n=45)$ with canine atopic dermatitis.

\begin{tabular}{cccc}
\hline Allergens & No. & Median & IR \\
\hline House dust mites & & & \\
Dermatophagoides pteronyssinus & 19 & 304 & $240-455$ \\
Dermatophagoides farinae & 31 & 935 & $373-2398$ \\
\hline Storage mites & & & \\
Lepidogliphus destructor & 17 & 335 & $210-562$ \\
Tyrophagus putrescentiae & 32 & 678 & $353-1778$ \\
Acarus siro & 32 & 640 & $270-2060$ \\
Malassezia & 11 & 273 & $219-574$ \\
\hline Molds & & & \\
Penicillium & 4 & 223 & $168-332$ \\
Alternaria alternata & 5 & 186 & $226-335$ \\
Aspergillus fumigatus & 6 & 3375 & No \\
\hline Fleas & 1 & No IR
\end{tabular}

EAU, ELISA Absorbance Units; IN, indoor allergen panel; IR, interquartile range.

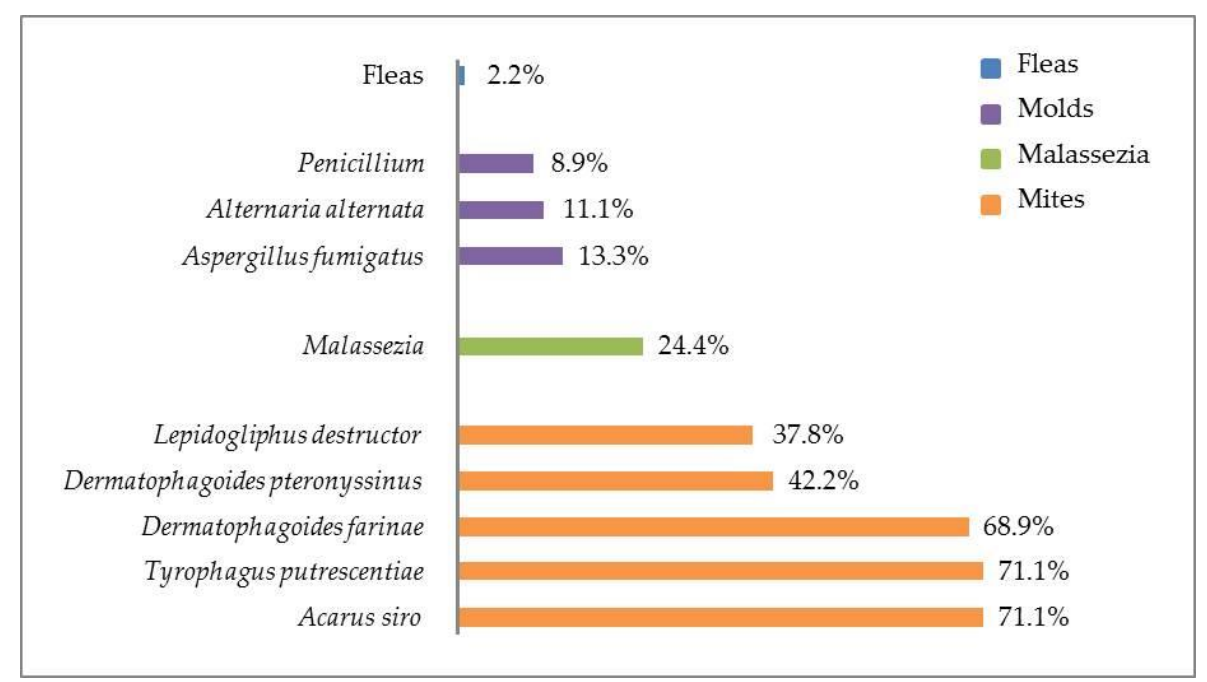

Figure 1. Distribution and percentage of various allergens in indoor allergen panel (fleas, molds, Malassezia, and mites) positive subjects $(n=45)$ with canine atopic dermatitis.

Frequent co-positives in the category of mites was found (Table 3). High co-positivity $(>70 \%)$ was observed among Dermatophagoides farinae and Acarus siro, Dermatophagoides farinae and Tyrophagus putrescentiae, and Tyrophagus putrescentiae and Acarus siro. 
Table 3. Number and (\%) of co-positives in the category of mites in IN positive subjects $(n=45)$ with CAD.

\begin{tabular}{cccccc}
\hline \multicolumn{1}{c}{ Allergens } & $\begin{array}{c}\text { Dermatophagoides } \\
\text { farinae }\end{array}$ & $\begin{array}{c}\text { Dermatophagoides } \\
\text { pteron. }\end{array}$ & Acarus siro & $\begin{array}{c}\text { Tyrophagus } \\
\text { putrescentiae }\end{array}$ & $\begin{array}{c}\text { Lepidogliphus } \\
\text { destructor }\end{array}$ \\
\hline Dermatophagoides farinae & & $22(48.9 \%)$ & $34(75.6 \%)$ & $33(73.3 \%)$ & $19(42.2 \%)$ \\
Dermatophagoides pteron. & $22(48.9 \%)$ & & $23(51.1 \%)$ & $22(48.9 \%)$ & $16(35.6 \%)$ \\
Acarus siro & $34(75.6 \%)$ & $23(51.1 \%)$ & $33(73.3 \%)$ & $33(73.3 \%)$ & $16(35.6 \%)$ \\
Tyrophagus putrescentiae & $33(73.3 \%)$ & $22(48.9 \%)$ & $16(35.6 \%)$ & $18(40.0 \%)$ & $18(40.0 \%)$ \\
Lepidogliphus destructor & $19(42.2 \%)$ & $16(35.6 \%)$ & & \\
\hline
\end{tabular}

$\mathrm{IN}$, indoor allergen panel; CAD, canine atopic dermatitis.

All OUT positive subjects ( $n=30,61.2 \%)$ showed positivity for at least one allergen of pollen from grasses, 28 for at least one allergen of pollen from weeds and 21 for at least one allergen of pollen from trees.

Serum IgE concentrations and distribution of various allergens in OUT positive subjects is reported in Table 4 and Figure 2, respectively.

Table 4. Serum IgE concentrations (EAU) of various allergens in OUT positive subjects $(n=30)$ with canine atopic dermatitis.

\begin{tabular}{cccc}
\hline Allergens & No. & Median & IR \\
\hline Grasses & & & $335-1011$ \\
Poa pratendis & 17 & 448 & $307-978$ \\
Dactylis glomerata & 19 & 508 & $270-1086$ \\
Lolium perenne & 20 & 421 & $214-811$ \\
Phleum pretense & 21 & 387 & $293-1111$ \\
Cynodon dactylon & 24 & 494 & $327-3500$ \\
\hline Weeds & & & $252-1427$ \\
Taraxacum vulgare & 5 & 827 & $211-776$ \\
Artemisia vulgaris & 8 & 479 & $205-414$ \\
Parietaria spp. & 12 & 277 & $297-552$ \\
Chenopodium álbum & 14 & 310 & $278-875$ \\
Plantago lanceolata & 15 & 435 & $172-215$ \\
Rumex acetosa & 23 & 524 & $197-441$ \\
\hline Trees & & & $176-586$
\end{tabular}

EAU, ELISA Absorbance Units; OUT, outdoor allergen panel; IR, interquartile range.

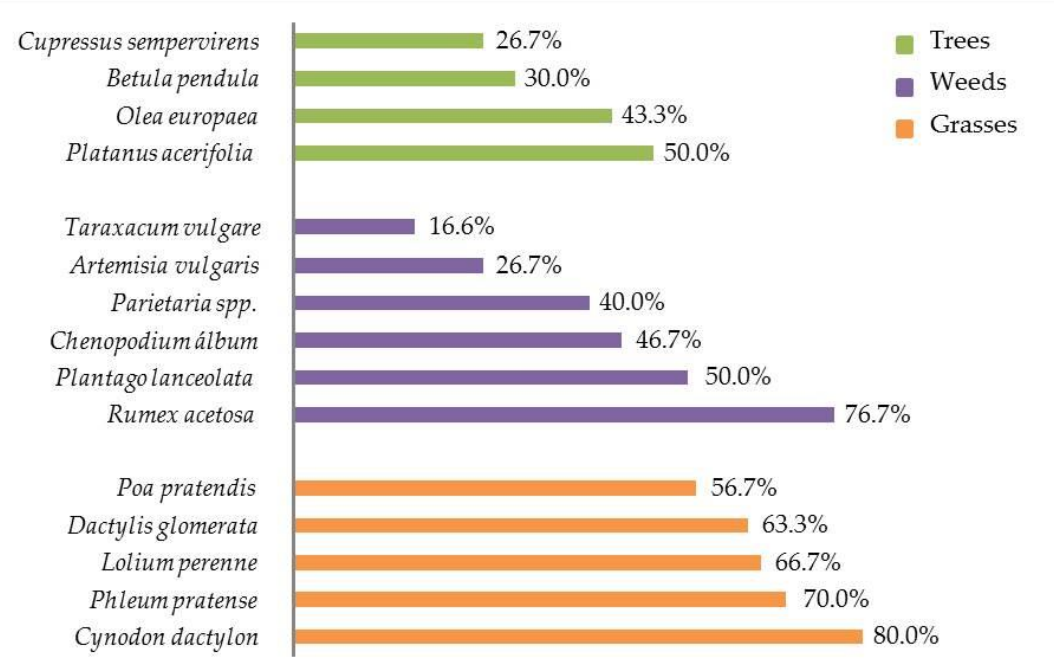

Figure 2. Distribution and percentage of various allergens in outdoor allergen panel (trees, weeds, and grasses) positive subjects $(n=30)$ with canine atopic dermatitis. 


\section{Discussion}

Despite the widespread use of SAT in clinical practice, the detection of IgE levels for specific allergens involved in CAD using this method has not been studied in dogs from Italy. Only a few studies using IDT are reported in Italy $[27,28]$, whereas there are various international studies from different geographical areas $[6,12,15,32-35]$ that have investigated SAT in CAD.

Our study was performed in Northern Italy and $71 \%$ of the subjects tested were positive for a screening serum allergy test for indoor and/or outdoor allergens. The proportion of CAD cases positive to SSAT reported in different international studies ranged from $74.4 \%$ to $85.4 \%[6,21,36,37]$. The actual prevalence of elevated IgE levels in dogs is not known $[10,38]$. The high proportion of positive results found in some studies is explained by the fact that the serum samples were submitted for IgE analysis only after clinical diagnosis of CAD was determined. In the present study, we found a lower rate of positivity compared with the aforementioned studies $[6,21,36,37]$. These results may be related to many factors such as the lower number of case studies, the type and number of allergen included in the serology panel, and the lack of performing IDT in addition to SAT to increase the diagnostic sensitivity [36,37], although there is no evidence that suggests that both need to be necessarily performed $[7,10]$. The percentage of CAD subjects positive to SAT reported in the present study is also lower than those found in the other studies conducted in Italy, although these studies are performed by using IDT [27,28]. These two testing methods are very different and a poor correlation of results between them is seen [7]; SAT measures circulating allergen-specific IgE and does not take into account other allergic pathways $[2,39]$ while IDT is an indirect measure of cutaneous mast cell reactivity due to the presence of IgE [2].

Several assays for allergen-specific IgE serology testing, mostly based on ELISAs, have been used both in human and in veterinary medicine. These assays are performed to detect specific IgE antibodies against a panel of allergens (e.g., pollen, mold, house dust mite, and epidermal allergens) considered relevant for the patient. In evaluating serum-based testing, the International Task force on CAD reported that the methodologies for these tests varies from laboratories; few critical studies have evaluated the performance of these tests [10]. In the past decades, the detection of serum $\operatorname{IgE}$ was done using monoclonal, mixed monoclonal, or polyclonal anti-canine IgE. However, due to the higher sensitivity and specificity of a monoclonal antibody, the use of polyclonal anti-canine IgE antibodies has markedly decreased $[10,40]$. Good results have been demonstrated with the veterinary assay using a unique recombinant fragment of the extracellular portion of the human high affinity $\operatorname{IgE}$ receptor alpha-subunit $(\mathrm{Fc \varepsilon RI} \alpha)$. It has shown a strong affinity for canine IgE other than a lack of cross-reactivity with the IgG [41,42]. Recently, the performance characteristics of a monoclonal antibody cocktail-based ELISA test as screening test for detection of allergen specific IgE in dogs (macELISA) has been thoroughly studied. High interlaboratory reproducibility and consistency of macELISA results have been documented between different laboratories [30,31]. Particularly, the variability between and among the European laboratories seem to be indistinguishable demonstrating that all laboratories are equally proficient in providing consistent results for all allergens tested. Moreover, the results from the macELISA are also directly comparable to those obtained with the assay using the alpha chain of human high affinity IgE receptor (Fc_R1; FceELISA) [42]. The macELISA assay has also demonstrated to have an adequate uniformity of manufactured lots coated wells and of each new lot of allergen extract that is to be used in the well manufacturing process. For the aforementioned reasons, we decided to use the macELISA method since all the variables are well controlled and the laboratory that performed the analysis in our study was one of those included in the recent study that validated the intra and inter-laboratory reproducibility of the assay [31]. For the macELISA, a cut-off of 150 EAU has demonstrated to establish $99 \%$ confidence for positive responses, whereas the variability of this test is most prominent and of clinical relevance with low values that are around the cut-off point [31]. In our study, a few cases in the molds category resulted 
in being slightly below the cut off (between 100 and 150 EAU). This could question the negative test results obtained in the study.

Differentiating between positive and negative responses to SSAT is of fundamental importance for therapeutic purposes; it aims in selecting allergen extract to be included in an immunotherapeutic regimen $[10,43,44]$. It should be noted that these ELISA results are not quantitative but semiquantitative at best, and that the results are usually interpreted as qualitative only. There is no compelling evidence that the level of allergen specific $\operatorname{IgE}$ correlates with severity of clinical disease [10].

The results of serological IgE tests may vary markedly due to the geographical differences in exposure to different allergens and the lack of a universal standardization of allergens extracts. The appropriate selection of allergens to test is fundamental to obtain reliable SAT results. In fact, allergens, mainly pollens, are subject to a great geographic variability. Thus, it is important for veterinarians performing SAT to identify the allergens present in the regional location where the patients live. Information about relevant allergens can be obtained by consulting National Allergy Bureau (http: / / www.worldallergy.org/pollen/) [11] It is of great clinical importance to update the allergen list based on climatic changes.

In our study, $29 \%$ of dogs tested showed a negative sSAT. These subjects might represent the CAD-like cases. In these cases, although patients present the same clinical signs of CAD, an IgE response to environmental or other allergens cannot be documented [4]. The real incidence of CAD-like is unknown [45]. In a retrospective study, Prelaud and Cochet-Faivre have obtained a CAD-like incidence percentage of $25.6 \%$. In a more recent study, a percentage of $14,6 \%$ was found [36,37]. In dogs, the differentiation between CAD and CAD-like is usually made on the basis of the results of IDT or SAT [46]. Indeed, IDT and/or SAT support the definitive diagnosis identifying an IgE response [30]. As previously mentioned, the lack of performing IDT in addition to SAT, might explain the higher percentage obtained in the present study. Moreover, false negative results may also be explained by the involvement of untested allergens or due to low IgE levels that are below the cut-off point of test and therefore to a low sensitivity of the sSAT which is currently unknown.

In our study, we found that the concentrations of $\operatorname{IgE}$ of allergens in IN positive subjects showed no difference compared with OUT positive subjects.

Among the positive screening tests, $91.8 \%$ were positive for IN (includes both only IN and IN plus OUT) and 61.2\% were positive for OUT (includes both only OUT and OUT plus IN). The high percentage of IN found in the present study is similar to another study conducted in Northern Europe [6]. These data can be explained by the high percentage of house dust mites positivity, which seems to represent the most important allergen involved in CAD in Europe [14].

Most of tested subjects in the present study are positive for more than one allergen and a high frequency (53\%) for both indoor and outdoor allergens. This result overlaps with the studies previously carried out in Italy and in Europe and confirms that also in our area the phenomenon of polysensitization exists [6,12,24,27,28,33,46-49]. Interestingly, this was found both among the allergens present within the same panel, and particularly, mostly between house dust and storage mites, and between those present in the two different panels.

In the IN positive subjects, according to previous studies conducted in Italy and in other European countries, mites are the group of allergens that give the most positive results in allergological tests $[17,19,23,27,33,50]$. In temperate climates, CAD is predominantly associated with Dermatophagoides species [35,51]. Our data showed a high positivity in both storage mites and house dust mites groups. Particularly, Dermatophagoides farinae, Tyrophagus putrescentiae, and Acarus siro were the most represented mites. The percentage of positivity found for Dermatophagoides farinae (68.9\%) was similar to those found in other Italian and European studies $[14,19,23,27,28,33,50]$, whereas the sensitization rate for Tyrophagus putrescentiae and Acarus siro was higher than previous Italian investigations $[27,28]$. On the other hand, the sensitization to storage mites found in our study agree with that reported 
in other European studies [6,35]. In the present study, a high co-positivity was observed among these three mites $(>73.0 \%)$. Studies in dogs have revealed extensive cross-reactions between house dust and storage mites [46,52-54]. This high co-positivity rate could be due to cross-reactions related to the SAT method used [35] or to a greater predisposition towards storage mite allergens in the geographical area analyzed in the present study.

In our results, Malassezia and molds antigens have shown a percentage of positivity of $24,4 \%$ and $26,7 \%$, respectively. It is well established that Malassezia pachydermatis is part of the normal cutaneous microbiota of dogs, although differing numbers of yeast occur at different anatomic sites. Malassezia antigens have been demonstrated to elicit a hypersensitivity response in atopic dogs [14,55]. In the literature, only few studies consider Malassezia as an allergen, thus not included in the majority of reports as they pre-dated its availability. In the international studies that use SAT to detect Malassezia, the positivity rate that was found widely varies and ranges between $0 \%$ and $60 \%[15,21,26,56]$. It is interesting to note that in a recent study that evaluated both IDT and SAT, IDT showed a positivity for Malassezia of 24\%, whereas no positivity was found to SAT [21]. In Italy, only Furiani et al. reported a percentage of positivity to this allergen of $35 \%$ by using IDT [27].

The importance of molds in atopic dogs is controversial. Previous reports demonstrate that sensitivity of the SAT to mold is lower than that of IDT, and SAT results are less reliable for detecting mold hypersensitivities [32,57]. Indeed, the positivity rate ranges between $0 \%$ and $44.8 \%[12,15,21,26,32]$. In our case series, we found several values slightly below the cut off (between 100 and 150 EAU) in molds category. It seems that the IgE levels in this category are likely to be low, therefore if a lower cut off is used the number of positive tests would consequently increase. Nevertheless, the percentage reactions to mold allergens could also be explained by the variations in temperature and humidity, typical of some seasons or geographical areas.

Interestingly, in our study, only one subject was positive to antigen's fleas. Likewise, other Italian and European studies have a medium-low incidence for this allergen [6,27,28,49]. In our study, these data can be justified as all examined subjects underwent a regular parasitic prophylaxis in their history and there was probably no sensitization to this allergen. However, since in the Greer antigen used for testing flea-specific IgE, is not extracted flea saliva but is crushed whole flea, negative reactions might simply result from a lack of sensitivity of the test.

In relation to positivity to outdoor allergens, it is difficult to make a comparison with other Italian and European studies, since, as previously stated, the distribution of pollen varies according to the geographical area and has varied over the years due to climate change. Regarding pollen, various factors such as geographical distributions and characteristics of plants, specific weather conditions, and season of the year must be considered.

In our study, data were collected throughout the years, thus comparing it with other Italian studies was difficult because either the studies tested different pollen or because a different method (IDT) was used. There was a high percentage of positivity to the OUT panel, which suggest that pollen may play a role in the development of CAD also in Italy. Particularly, pollen from grass showed a positivity of over $60 \%$ (Cynodon dactylon was the most represented). This was higher than those identified in other Italian studies where grasses showed a percentage of positive reactions < 30\% by using IDT $[27,28]$. As expressed above, it is not possible to compare SAT and IDT methodologies. It is not known whether IDT is under-recognizing pollen allergies (low sensitivity) and/or SAT is reporting pollen allergies (low specificity) [21].

Weed groups also showed a high positivity (57.1\%). Interestingly, Rumex acetosa (sorrel) was the only pollen of weeds that showed a high positivity $(76.7 \%)$ in the present study. Sorrel is a weed that belongs to the family of the Polygonaceae and grows spontaneously. It is present in all regions of Italy, and blooms between May and August. There are no data on this allergen in other studies conducted in Italy. However, it is interesting to note that in a recent study conducted in Norway, a plant belonging to the genus Rumex (Rumex acetosella) 
was included in an allergy test and was the major positive allergen, with an incidence of $40 \%$ [6]. Similarly, sorrel was the only pollen allergen that appeared in the top four reactions in both the IDT and SAT tests performed in a study in South Australia [21]. In addition, Barili et al. reported a positivity SAT of sorrel of 38\% [26]. In a recent Spanish study using SAT in horses with recurrent airway obstruction or atopic dermatitis, the most represented pollen allergens belonged to the genus Rumex [58]. The data relating to sensitization to this pollen obtained in the present study highlight the need to include this allergen in the outdoor panel also in Italy. Probably, the climate changes occurred over the last decade has allowed the spread of this pollen worldwide.

In this study, seropositivity to pollen of trees is found to be the lowest $(42.9 \%)$ in the OUT, but high when compared to the positivity found in other Italian studies that used IDT $[27,28]$. Comparison with international studies is not possible due to testing different plants. In humans, cross-reactions between tree pollens are generally only significant between species of the same genera [21]. No data are available on this aspect in veterinary medicine. As previously mentioned, sensitivity and specificity of IDT and SAT for pollens are controversial.

Although this is a preliminary study and therefore it is necessary to increase the case series, the results of the present report confirm that the appropriate selection of allergens to test is fundamental to obtain reliable SAT results and that a continuous update of possible new environmental allergens is necessary, especially for those allergens, mainly pollens, subject to a great geographic variability. Thus, it is important for veterinarians who perform SAT to identify the allergens present in the regional location where the patients live; type and number of allergen included in the serology panel need to be continually adapted to the specific geographical area.

\section{Conclusions}

This is the first study showing data on frequency of allergens involved in CAD in Northern Italy by using a well-established monoclonal antibody-based ELISA test for detection of allergen-specific IgE in dogs.

This study shows that a high IgE hypersensitivity in atopic dogs of Northern Italy was most often associated with indoor allergens, primarily house dust mites, while mold and flea saliva play a marginal role. Among the outdoor allergens, an important role was shown by Rumex acetosa, which had previously never been considered in Italy. Polysensitization also commonly occurs.

In accordance to the results in this study, specific panels for geographical areas should be considered and re-evaluated at time intervals considering the numerous factors affecting the prevalence of IgE positivity in atopic dogs, and the continuous changes of climate conditions, pollination periods in individual areas, weather conditions, variations in distributions of individual plants, environmental hygiene, and residential environments.

Author Contributions: Conceptualization, M.D.T. and F.R.; clinical and diagnostic procedures, M.B. and P.R.; dataset organization, A.L. and P.E.C.; writing-original draft preparation, M.D.T., F.R., M.B., and A.M.; writing-review and editing, M.D.T., F.R., A.M., A.L., and P.E.C.; supervision, M.D.T., F.R., and A.M. All authors have read and agreed to the published version of the manuscript.

Funding: This research received no external funding.

Institutional Review Board Statement: Ethical review and approval were not required for this study as all the animals admitted to the Veterinary Clinic, with consent from the owners, routinely undergo blood withdrawal and all the data were collected as part of the clinical investigations. Moreover, the animal care procedures were compliant to the national legislation on animal care (Legislation decree n.26, 03/03/2014) and to the European recommendations for the protection of animals used for clinical and scientific purposes (Directive 2010/63/EU), and adhered to the internal rules of University of Teramo.

Data Availability Statement: The data presented in this study are available on request from the corresponding authors. The data are not publicly available due to privacy protection. 
Conflicts of Interest: The authors declare no conflict of interest.

\section{References}

1. Halliwell, R.E.; DeBoer, D.J. The ACVD Task Force on Canine Atopic Dermatitis (III): The Role of Antibodies in Canine Atopic Dermatitis. Vet. Immunol. Immunopathol. 2001, 81, 159-167. [CrossRef]

2. Marsella, R.; Sousa, C.A.; Gonzales, A.J.; Fadok, V.A. Current Understanding of the Pathophysiologic Mechanisms of Canine Atopic Dermatitis. J. Am. Vet. Med. Assoc. 2012, 241, 194-207. [CrossRef] [PubMed]

3. Marsella, R.; De Benedetto, A. Atopic Dermatitis in Animals and People: An Update and Comparative Review. Vet. Sci. 2017, 4, 37. [CrossRef] [PubMed]

4. Halliwell, R. Revised Nomenclature for Veterinary Allergy. Vet. Immunol. Immunopathol. 2006, 114, 207-208. [CrossRef] [PubMed]

5. Hill, P.B.; DeBoer, D.J. The ACVD Task Force on Canine Atopic Dermatitis (IV): Environmental Allergens. Vet. Immunol. Immunopathol. 2001, 81, 169-186. [CrossRef]

6. Bjelland, A.A.; Dolva, F.L.; Nødtvedt, A.; Sævik, B.K. Prevalence of and Risk Factors for Increased Serum Levels of AllergenSpecific IgE in a Population of Norwegian Dogs. Acta Vet. Scand. 2014, 56, 81. [CrossRef]

7. Hensel, P.; Santoro, D.; Favrot, C.; Hill, P.; Griffin, C. Canine Atopic Dermatitis: Detailed Guidelines for Diagnosis and Allergen Identification. BMC Vet. Res. 2015, 11, 196. [CrossRef]

8. Pucheu-Haston, C.M.; Bizikova, P.; Eisenschenk, M.N.C.; Santoro, D.; Nuttall, T.; Marsella, R. Review: The Role of Antibodies, Autoantigens and Food Allergens in Canine Atopic Dermatitis. Vet. Dermatol. 2015, 26, 115-e30. [CrossRef]

9. DeBoer, D.J.; Hillier, A. The ACVD Task Force on Canine Atopic Dermatitis (XV): Fundamental Concepts in Clinical Diagnosis. Vet. Immunol. Immunopathol. 2001, 81, 271-276. [CrossRef]

10. DeBoer, D.J.; Hillier, A. The ACVD Task Force on Canine Atopic Dermatitis (XVI): Laboratory Evaluation of Dogs with Atopic Dermatitis with Serum-Based "Allergy" Tests. Vet. Immunol. Immunopathol. 2001, 81, 277-287. [CrossRef]

11. Hillier, A.; DeBoer, D.J. The ACVD Task Force on Canine Atopic Dermatitis (XVII): Intradermal Testing. Vet. Immunol. Immunopathol. 2001, 81, 289-304. [CrossRef]

12. Park, S.; Ohya, F.; Yamashita, K.; Nishifuji, K.; Iwasaki, T. Comparison of Response to Immunotherapy by Intradermal Skin Test and Antigen-Specific IgE in Canine Atopy. J. Vet. Med. Sci. 2000, 62, 983-988. [CrossRef] [PubMed]

13. Olivry, T.; Saridomichelakis, M. International Committee on Atopic Diseases of Animals (ICADA) Evidence-Based Guidelines for Anti-Allergic Drug Withdrawal Times before Allergen-Specific Intradermal and IgE Serological Tests in Dogs. Vet. Dermatol. 2013, 24, 225.e49. [CrossRef] [PubMed]

14. Miller, W.H.; Griffin, C.E.; Campbell, K.L. Hypersensitivity Disorders. In Muller and Kirk's Small Animal Dermatology, 7th ed.; Elsevier: St Louis, MO, USA; pp. 363-431.

15. Kang, M.-H.; Kim, H.-J.; Jang, H.-J.; Park, H.-M. Sensitization Rates of Causative Allergens for Dogs with Atopic Dermatitis: Detection of Canine Allergen-Specific IgE. J. Vet. Sci. 2014, 15, 545-550. [CrossRef] [PubMed]

16. Mueller, R.S.; Janda, J.; Jensen-Jarolim, E.; Rhyner, C.; Marti, E. Allergens in Veterinary Medicine. Allergy 2016, 71, 27-35. [CrossRef]

17. Vollset, I. Atopic Dermatitis in Norwegian Dogs. Nord. Vet. Med. 1985, 37, 97-106.

18. Mueller, R.S.; Bettenay, S.V.; Tideman, L. Aero-Allergens in Canine Atopic Dermatitis in Southeastern Australia Based on 1000 Intradermal Skin Tests. Aust. Vet. J. 2000, 78, 392-399. [CrossRef]

19. Sture, G.H.; Halliwell, R.E.; Thoday, K.L.; van den Broek, A.H.; Henfrey, J.I.; Lloyd, D.H.; Mason, I.S.; Ferguson, E. Canine Atopic Disease: The Prevalence of Positive Intradermal Skin Tests at Two Sites in the North and South of Great Britain. Vet. Immunol. Immunopathol. 1995, 44, 293-308. [CrossRef]

20. Masuda, K.; Sakaguchi, M.; Fujiwara, S.; Kurata, K.; Yamashita, K.; Odagiri, T.; Nakao, Y.; Matsuki, N.; Ono, K.; Watari, T.; et al Positive Reactions to Common Allergens in 42 Atopic Dogs in Japan. Vet. Immunol. Immunopathol. 2000, 73, 193-204. [CrossRef]

21. Han, C.; Chan, W.Y.; Hill, P.B. Prevalence of Positive Reactions in Intradermal and IgE Serological Allergy Tests in Dogs from South Australia, and the Subsequent Outcome of Allergen-Specific Immunotherapy. Aust. Vet. J. 2020, 98, 17-25. [CrossRef]

22. Youn, H.-Y.; Kang, H.-S.; Bhang, D.-H.; Kim, M.-K.; Hwang, C.-Y.; Han, H.-R. Allergens Causing Atopic Diseases in Canine. J. Vet. Sci. 2002, 3, 335-341. [CrossRef] [PubMed]

23. Saridomichelakis, M.N.; Koutinas, A.F.; Gioulekas, D.; Leontidis, L. Canine Atopic Dermatitis in Greece: Clinical Observations and the Prevalence of Positive Intradermal Test Reactions in 91 Spontaneous Cases. Vet. Immunol. Immunopathol. 1999, 69, 61-73. [CrossRef]

24. Chanthick, C.; Anaman, S.; Buathet, K. The Prevalence of Positive Intradermal Allergy Tests in 114 Dogs with Atopic Dermatitis in the Bangkok Metropolis, Thailand. Vet. Immunol. Immunopathol. 2008, 126, 256-262. [CrossRef] [PubMed]

25. Zur, G.; Ihrke, P.J.; White, S.D.; Kass, P.H. Canine Atopic Dermatitis: A Retrospective Study of 266 Cases Examined at the University of California, Davis, 1992-1998. Part I. Clinical Features and Allergy Testing Results. Vet. Dermatol. 2002, 13, 89-102. [CrossRef]

26. Barili, Ö.; Pekmezci, D. Samsun İli ve Çevresindeki Atopik Dermatitisli Köpeklerde Serum Spesifik IgE Tespiti ile Alerjen Tayini. Kocatepe Veteriner. Dergisi. 2019, 12, 413-423. [CrossRef]

27. Furiani, N.; Scarampella, F.; Noli, C.; Ordeix, L. A retrospective study of 486 intradermal tests performed on atopic dogs in Northern Italy. Veterinaria (Cremona) 2009, 23, 41-46. 
28. Tognetti, R.; Corazza, M.; Buonaccorsi, A. Considerazioni sui risultati dell'intradermoreazione in 230 cani atopici provenienti dalla regione Toscana. Veterinaria 1997, 11, 13-17.

29. Favrot, C.; Steffan, J.; Seewald, W.; Picco, F. A Prospective Study on the Clinical Features of Chronic Canine Atopic Dermatitis and Its Diagnosis. Vet. Dermatol. 2010, 21, 23-31. [CrossRef]

30. Lee, K.W.; Blankenship, K.D.; McCurry, Z.M.; Esch, R.E.; DeBoer, D.J.; Marsella, R. Performance Characteristics of a Monoclonal Antibody Cocktail-Based ELISA for Detection of Allergen-Specific IgE in Dogs and Comparison with a High Affinity IgE Receptor-Based ELISA. Vet. Dermatol. 2009, 20, 157-164. [CrossRef]

31. Lee, K.W.; Blankenship, K.D.; McCurry, Z.M.; McKinney, B.; Ruffner, R.; Esch, R.E.; Tambone, C.; Faas, R.; Hermes, D.; Brazis, P.; et al. Intra and Inter-Laboratory Reproducibility of a Monoclonal Antibody Cocktail Based ELISA for Detection of Allergen Specific IgE in Dogs: Proficiency Monitoring of MacELISA in Six Laboratories. Vet. Immunol. Immunopathol. 2012, 148, 267-275. [CrossRef]

32. Foster, A.P.; Littlewood, J.D.; Webb, P.; Wood, J.L.N.; Rogers, K.; Shaw, S.E. Comparison of Intradermal and Serum Testing for Allergen-Specific IgE Using a Fcepsilon RIalpha-Based Assay in Atopic Dogs in the UK. Vet. Immunol. Immunopathol. 2003, 93, 51-60. [CrossRef]

33. Goicoa, A.; Espino, L.; Rodriguez, I.; Puigdemont, A.; Brazis, P.; Rejas, J. Importance of House Dust and Storage Mites in Canine Atopic Dermatitis in the Geographic Region of Galicia, Spain. Acta Vet. Hung. 2008, 56, 163-171. [CrossRef] [PubMed]

34. Popiel, J.; Cekiera, A. Comparison of IgE Test Results with Intradermal Skin Tests for Dust Mites and Storage Mites in Atopic Dogs. Pol. J. Vet. Sci. 2015, 18, 351-356. [CrossRef] [PubMed]

35. Arlian, L.G.; Schumann, R.J.; Morgan, M.S.; Glass, R.L. Serum Immunoglobulin E against Storage Mite Allergens in Dogs with Atopic Dermatitis. Am. J. Vet. Res. 2003, 64, 32-36. [CrossRef] [PubMed]

36. Botoni, L.S.; Torres, S.M.F.; Koch, S.N.; Heinemann, M.B.; Costa-Val, A.P. Comparison of Demographic Data, Disease Severity and Response to Treatment, between Dogs with Atopic Dermatitis and Atopic-like Dermatitis: A Retrospective Study. Vet. Dermatol. 2019, 30, 10.e4. [CrossRef]

37. Preloud, P.; Cochet-Faivre, N. A retrospective study of 21 cases of canine atopic-like dermatitis. 22nd Annual Congress of the ESVD-ECVD, 13-15 September 2007, Mainz, Germany. Vet. Dermatol. 2007, 18, 384-388. [CrossRef]

38. Hillier, A.; Griffin, C.E. The ACVD Task Force on Canine Atopic Dermatitis (I): Incidence and Prevalence. Vet. Immunol. Immunopathol. 2001, 81, 147-151. [CrossRef]

39. Hensel, P.; Bauer, C.L.; Austel, M.; Keys, D. Serological and intradermal test reactivity patterns among six species of house dust and storage mites. NAVDF 2009 Abstracts. Vet. Dermatol. 2009, 20, 228-229. [CrossRef]

40. Saevik, B.K.; Ulstein, T.L.; Larsen, H.J.S. Evaluation of a Commercially Available Enzyme-Linked Immunosorbent Assay for the Detection of Allergen-Specific IgE Antibodies in Dogs. Res. Vet. Sci. 2003, 74, 37-45. [CrossRef]

41. Wassom, D.L.; Grieve, R.B. In Vitro Measurement of Canine and Feline IgE: A Review of FceR1 $\alpha$-Based Assays for Detection of Allergen-Reactive IgE. Vet. Dermatol. 1998, 9, 173-178. [CrossRef]

42. Stedman, K.; Lee, K.; Hunter, S.; Rivoire, B.; McCall, C.; Wassom, D. Measurement of Canine IgE Using the Alpha Chain of the Human High Affinity IgE Receptor. Vet. Immunol. Immunopathol. 2001, 78, 349-355. [CrossRef]

43. Olivry, T.; Sousa, C.A. The ACVD Task Force on Canine Atopic Dermatitis (XIX): General Principles of Therapy. Vet. Immunol. Immunopathol. 2001, 81, 311-316. [CrossRef]

44. Griffin, C.E.; Hillier, A. The ACVD Task Force on Canine Atopic Dermatitis (XXIV): Allergen-Specific Immunotherapy. Vet. Immunol. Immunopathol. 2001, 81,363-383. [CrossRef]

45. Santoro, D. Therapies in Canine Atopic Dermatitis: An Update. Vet. Clin. N. Am. Small Anim. Pract. 2019, 49, 9-26. [CrossRef]

46. Buckley, L.; Schmidt, V.; McEwan, N.; Nuttall, T. Cross-Reaction and Co-Sensitization among Related and Unrelated Allergens in Canine Intradermal Tests. Vet. Dermatol. 2013, 24, 422-427. [CrossRef]

47. Bensignor, E.; Carlotti, D.N. Sensitivity Patterns to House Dust Mites and Forage Mites in Atopic Dogs: 150 Cases. Vet. Dermatol. 2002, 13, 37-42. [CrossRef]

48. Tarpataki, N.; Pápa, K.; Reiczigel, J.; Vajdovich, P.; Vörösi, K. Prevalence and Features of Canine Atopic Dermatitis in Hungary. Acta Vet. Hung. 2006, 54, 353-366. [CrossRef]

49. Noli, C.; Candian, M.; Scarpa, P. Analisi di una casistica specialistica dermatologica del nord Italia: 1188 casi (1995-2002). Veterinaria 2006, 20, 39-50.

50. Carlotti, D.N.; Costargent, F. Analysis of positive skin tests in 449 dogs with allergic dermatitis. Eur. J. Companion Anim. Pract. 1994, 44, 293-308.

51. Randall, A.; Hillier, A.; Cole, L.K.; Kwochka, K.W.; Needham, G.; Wassom, D.L. Quantitation of House Dust Mites and House Dust Mite Allergens in the Microenvironment of Dogs. Am. J. Vet. Res. 2003, 64, 1301-1309. [CrossRef]

52. Nuttall, T.J.; Hill, P.B.; Bensignor, E.; Willemse, T. members of the International Task Force on Canine Atopic Dermatitis House Dust and Forage Mite Allergens and Their Role in Human and Canine Atopic Dermatitis. Vet. Dermatol. 2006, 17, $223-235$. [CrossRef] [PubMed]

53. Saridomichelakis, M.N.; Marsella, R.; Lee, K.W.; Esch, R.E.; Farmaki, R.; Koutinas, A.F. Assessment of Cross-Reactivity among Five Species of House Dust and Storage Mites. Vet. Dermatol. 2008, 19, 67-76. [CrossRef] [PubMed]

54. Marsella, R.; Saridomichelakis, M.N. Environmental and Oral Challenge with Storage Mites in Beagles Experimentally Sensitized to Dermatophagoides Farinae. Vet. Dermatol. 2010, 21, 105-111. [CrossRef] [PubMed] 
55. Farver, K.; Morris, D.O.; Shofer, F.; Esch, B. Humoral Measurement of Type-1 Hypersensitivity Reactions to a Commercial Malassezia Allergen. Vet. Dermatol. 2005, 16, 261-268. [CrossRef]

56. Ishimaru, H.; Okamoto, N.; Fujimura, M.; Miyaji, K.; Shimakura, H.; Takase, Y.; Mizukami, K.; Uchiyama, J.; DeBoer, D.J.; Sakaguchi, M. IgE Sensitivity to Malassezia Pachydermatis and Mite Allergens in Dogs with Atopic Dermatitis. Vet. Immunol. Immunopathol. 2020, 226, 110070. [CrossRef]

57. Mueller, R.S.; Burrows, A.; Tsohalis, J. Comparison of Intradermal Testing and Serum Testing for Allergen-Specific IgE Using Monoclonal IgE Antibodies in 84 Atopic Dogs. Aust. Vet. J. 1999, 77, 290-294. [CrossRef]

58. Marteles, D.; Odriozola, L.; Verde, M.T.; Conde, T.; Fernández, A. Assessment of Serum Allergen-Specific IgE Levels in Horses with Seasonal Allergic Dermatitis and Recurrent Airway Obstruction in Spain. Acta Vet. Hung. 2019, 67, 11-21. [CrossRef] 